\title{
The Effects of Postprandial Resistance Exercise on Blood Glucose and Lipids in Prediabetic, Beta-Thalassemia Major Patients
}

\author{
Kalliopi Georgakouli ${ }^{1,2}{ }^{\oplus}$, Alexandra Stamperna ${ }^{1}\left(\right.$, Chariklia K. Deli ${ }^{1}{ }^{\oplus}$, Niki Syrou ${ }^{1}{ }^{\oplus}$, \\ Dimitrios Draganidis ${ }^{1}$ (D), Ioannis G. Fatouros ${ }^{1}$ and Athanasios Z. Jamurtas ${ }^{1, *(D)}$ \\ 1 Department of Physical Education and Sport Science, University of Thessaly, Karies, 42100 Trikala, Greece; \\ kgeorgakouli@gmail.com (K.G.); alexstaberna@gmail.com (A.S.); delixar@pe.uth.gr (C.K.D.); \\ nikisyrou@uth.gr (N.S.); dimidraganidis@gmail.com (D.D.); ifatouros@uth.gr (I.G.F.) \\ 2 Department of Nutrition \& Dietetics, University of Thessaly, Karies, 42100 Trikala, Greece \\ * Correspondence: ajamurt@pe.uth.gr; Tel.: +30-243-104-7054
}

Received: 29 January 2020; Accepted: 21 April 2020; Published: 26 April 2020

check for updates

\begin{abstract}
Insulin resistance and diabetes mellitus are common consequences of iron overload in the pancreas of beta-thalassemia major (BTM) patients. Moreover, postprandial blood glucose elevations are linked to major vascular complications. The purpose of this study was to investigate the effects of a bout of acute resistance exercise following breakfast consumption of glucose and fat on the metabolism in prediabetic, BTM patients. Six patients underwent two trials (exercise and control) following breakfast consumption (consisting of approximately $50 \%$ carbohydrates, $15 \%$ proteins, 35\% fat), in a counterbalanced order, separated by at least three days. In an exercise trial, patients performed chest and leg presses ( 3 sets of 10 repetitions maximum/exercise), while in the control trial they rested. Blood samples were obtained in both trials at: pre-meal, 45 min post-meal (pre-exercise/control), post-exercise/control, $1 \mathrm{~h}$ post-exercise/control, $2 \mathrm{~h}$ post-exercise/control and $24 \mathrm{~h}$ post-exercise/control. Blood was analysed for glucose and lipids (total cholesterol, High Density Lipoprotein-cholesterol, Low Density Lipoprotein-cholesterol, triglycerides). Blood glucose levels increased significantly $45 \mathrm{~min}$ following breakfast consumption. Blood glucose and lipids did not differ between trials at the same time points. It seems that a single bout of resistance training is not sufficient to improve blood glucose and fat levels for the subsequent 24-h post-exercise period in prediabetic, BTM patients.
\end{abstract}

Keywords: haemoglobin; diabetes; fitness; cardiovascular health; nutrition

\section{Introduction}

Prediabetes is as a state of intermediate hyperglycaemia where a person has impaired fasting glucose, impaired glucose tolerance or a combination of the two [1]. Although glucose levels in prediabetes are not yet high enough for a diagnosis of Diabetes Mellitus (DM), prediabetes is associated with macrovascular and microvascular complications of DM, including nephropathy, small fibre neuropathy, retinopathy and coronary artery disease. Moreover, individuals with prediabetes are at high risk of developing DM [2].

Lifestyle interventions have been shown to reduce the risk of DM in adults with prediabetes, and they should be an essential part of the management of this condition. The main problem in glycaemic control is the peak of glucose 1-2 h after a meal, i.e., postprandial hyperglycaemia. Exercise increases contraction-mediated glucose uptake resulting in reduced postprandial hyperglycaemia and has been proposed as an effective way to improve glucose control in individuals with type 2 DM. Indeed, 
the timing of exercise relative to meal consumption may play a role in glycaemic control. The limited available data indicate that postprandial (consumption following dinner) exercise may be more beneficial than preprandial exercise in type $2 \mathrm{DM}$ patients, and both aerobic and resistance training have been shown to be effective [3]. In addition, postprandial resistance exercise improves triglyceride levels, another risk factor for cardiovascular disease in type 2 DM [4]. Thus, postprandial resistance exercise may be an effective means of better glycaemic control and a lower risk of cardiovascular disease in individuals with an abnormal glucose metabolism. The optimal postprandial exercise timing and prescription are yet to be defined.

Beta-thalassemia major (BTM) is an inherited haemoglobin disorder that manifests within the first few months of life with ineffective erythropoiesis and chronic haemolytic anaemia, and frequent blood transfusions are required. There is no physiological mechanism to remove the excess iron load resulting from regular blood transfusions, while ineffective erythropoiesis increases intestinal iron absorption. Both processes induce iron accumulation in reticuloendothelial cells and parenchymal tissues that can cause progressive damage in multiple organs [5]. Iron accumulation in the pancreatic islets induces insulin resistance and reduced early insulin secretion, often resulting in DM in BTM patients [6]. Although the pathophysiological mechanisms leading to the development of DM are still unclear, it is most likely linked to the reversible oxidation and reduction of iron. This property renders iron potentially hazardous due to its ability to participate in the generation of reactive oxygen species [7], while pancreatic islets are susceptible to oxidative damage as they almost exclusively rely on the mitochondrial metabolism of glucose for glucose-induced insulin secretion and they also have a low antioxidant defence system [8]. Exercise is thought to provide various beneficial health effects in various metabolic disorders; however, research on the effects of exercise in BTM patients is non-existent. This could be due to the fact that BTM patients often manifest exercise intolerance and fatigue mediated by anaemia and iron-mediated cardiotoxicity [9].

This study was therefore designed to investigate whether postprandial resistance exercise can influence changes in glucose and lipid metabolism in prediabetic, BTM patients. Based on previous literature on the effects of postprandial exercise on health parameters in prediabetics, we hypothesized that an acute bout of resistance exercise $45 \mathrm{~min}$ following breakfast consumption would attenuate the blood glucose response and improve lipid profiles throughout the subsequent 24 -h post-exercise period.

\section{Materials and Methods}

\subsection{Participants}

Six prediabetic, BTM patients ( 3 men, 3 women; age: $39.5 \pm 4.6$ years) volunteered to participate in this study. All of them had their doctor's permission to participate, were informed about the study protocol, filled a medical history questionnaire and signed an informed consent form. Due to transfusions, these patients had suppressed their autologous hematopoiesis. Haemoglobin in BTM patients comes mainly from frequent transfusions and therefore has a normal $\mathrm{O} 2$ affinity. Procedures were in accordance with the 1975 Declaration of Helsinki (2000) and approval was obtained from the Institutional Review Board of the Department of Physical Education and Sport Science, University of Thessaly (protocol number 1076). Moreover, the study was registered at ClinicalTrials.gov as NCT03889977 [10].

Inclusion criteria:

- $\quad$ BTM patients requiring regular blood transfusions.

- Confirmed prediabetes with patients fulfilling one if the three following criteria: (a) impaired fasting glucose (IFG) (fasting plasma glucose (FPG) of 6.1-6.9 mmol/L), or (b) impaired glucose tolerance (IGT) (plasma glucose of 7.8-11.0 mmol/L, $2 \mathrm{~h}$ following ingestion of $75 \mathrm{~g}$ of oral glucose load) or (c) a combination of the two, based on a $2 \mathrm{~h}$ oral glucose tolerance test [1].

- $\quad$ Age group of 25 to 55 .

Exclusion criteria: 
- Hypertension.

- Injuries.

- Any other serious complications of BTM that could contraindicate participation to exercise.

Moreover, volunteers were instructed to avoid lifestyle changes and strenuous or unusual physical activity for at least two days before each visit to the laboratory.

\subsection{Experimental Design}

All participants reported to the laboratory in the morning (9:00-10:00 a.m.) for physiological measurements (blood pressure, resting heart rate, body composition analysis through DEXA test) and determination of one repetition maximum (1RM). Moreover, they were asked to record their diet for two days before their next visit and followed the same diet before the third and last visit.

In a randomized within-subject design, participants underwent two trials (exercise, ExT and control, CoT) $45 \mathrm{~min}$ following breakfast consumption (consisting of approximately $50 \%$ carbohydrates, $15 \%$ proteins, $35 \%$ fat), in a counterbalanced order, separated by at least three days.

In each one of the experimental trials, participants reported to the laboratory in the morning (9:00-10:00 a.m.) after overnight fasting ( 10 h) and a blood sample was drawn. Then they were provided with a standard breakfast that they had to consume in $10 \mathrm{~min}$. Forty-five min later, another blood sample was drawn. In the ExT, the participants initially performed a warm-up for 5-10 min consisting of 8-10 repetitions using a light weight. Then they performed chest and leg press exercises ( 3 sets of 10 repetitions at 70\% of their 1RM, each), and they finished the workout by performing stretching exercises for approximately $5 \mathrm{~min}$ of the two major muscle groups used during the workout. In the CoT, participants rested for the same duration. Blood samples were obtained immediately after ExT and CoT, as well as $1 \mathrm{~h}, 2 \mathrm{~h}$ and $24 \mathrm{~h}$ following each trial. Water consumption throughout trials was ad libitum.

\subsection{Anthropometric and Physiological Characteristics}

Body height was measured with a precision of $0.1 \mathrm{~cm}$ and body weight with a precision of $0.1 \mathrm{~kg}$ (Beam Balance, Seca, Birmingham, UK), with the participants lightly dressed and barefoot. Body fat percentage was estimated by dual-energy X-ray absorptiometry (DEXA) (Lunar DPX NT, GE Healthcare, Chalfont St. Giles, Buckinghamshire, UK). Blood pressure (BP) was measured with a manual sphygmomanometer (FC-101 Aneroid Sphygmomanometer; Focal Corporation, Kashiwa, Japan) after 5 min of seated rest.

\subsection{Blood Collection and Handling}

In total, blood samples were obtained from a forearm vein with participants in a seated position at the following time-points: pre-meal, $45 \mathrm{~min}$ post-meal (pre-ExT/CoT), immediately post- ExT/CoT, $1 \mathrm{~h}$ post-ExT/CoT, $2 \mathrm{~h}$ post-ExT/CoT, $24 \mathrm{~h}$ post- ExT/CoT. Blood samples were obtained after $10 \mathrm{~min}$ rest, except for immediately post-ExT, where blood was obtained immediately following exercise. Blood was immediately transferred to a tube containing Ethylene diamine tetraacetic acid (EDTA) and centrifuged in order to obtain the plasma which was stored in aliquots at $-80{ }^{\circ} \mathrm{C}$ until the day of analysis. Plasma preparation has been described elsewhere [11].

\subsection{Blood Analysis}

Samples underwent only one freeze-thaw cycle and each parameter was measured in duplicates. Plasma glucose and lipids (total cholesterol, HDL-cholesterol, LDL-cholesterol, triglycerides) were determined using a biochemical analyser (Clinical Chemistry Analyzer Z1145; Zafiropoulos Diagnostica S.A., Koropi, Greece). LDL-cholesterol was calculated using the Friedewald equation [12]. 


\subsection{Statistical Analysis}

Preliminary power analysis performed with $G^{*}$ Power [13] showed that the minimum required sample size was 6 (with a probability error of 0.05 , a statistical power of $80 \%$ and an effect size of 0.5 ).

Normality was checked using the Shapiro-Wilk test. Since some variables did not follow normal distribution, nonparametric statistics were used for the analyses. The Friedman analysis of variance by ranks test was performed to determine the time effects, accompanied by the Wilcoxon signed-rank test to perform pairwise comparisons. Differences between trials were examined using the Mann-Whitney $\mathrm{U}$ test (U values). Differences between genders for areas under the curve (AUC) and triglycerides was assessed by a $2 \times 2$ (gender by condition) ANOVA. The level of statistical significance was set at $p<0.05$. Data are presented as means \pm SD. Statistical analysis was conducted with IBM SPSS Version 19.0 (IBM Corp., Armonk, NY, USA).

Moreover, the incremental areas under the curves (AUCs) were measured for plasma glucose and triglycerides with GraphPad Prism version 5.0 (GraphPad Software, San Diego, CA, USA). Differences in AUCs between trials were examined using the Mann-Whitney U test (U values).

\section{Results}

\subsection{Anthropometric and Physiological Characteristics}

Anthropometric and physiological characteristics of the participants did not differ between the ExT and CoT (Table 1). The mean body mass index (BMI) indicated that men were overweight and women were normal weight. According to the International Diabetes Federation, the ethnic group specific cut-point for waist circumference (WC) is $94 \mathrm{~cm}$ and $80 \mathrm{~cm}$ for European men and women, respectively [14]. WC of the participants in the present study exceeded this cut-point, indicating central obesity.

Table 1. Anthropometric and physiological characteristics before exercise (ExT) and control trial (CoT).

\begin{tabular}{|c|c|c|}
\hline Variable & ExT & CoT \\
\hline $\mathrm{BM}(\mathrm{kg})$ & $66.0 \pm 16.6$ & $65.5 \pm 16.2$ \\
\hline BMI $\left(\mathrm{kg} / \mathrm{m}^{2}\right)$ & $\begin{array}{c}24.2 \pm 5.4 \text { (men: } 25.7 \pm 7.2 \text {; women: } 22.6 \\
\pm 3.8 \text { ) }\end{array}$ & $\begin{array}{c}24.0 \pm 5.2 \text { (men: } 25.6 \pm 6.8 \text {; women: } 22.4 \\
\pm 3.7 \text { ) }\end{array}$ \\
\hline$\% \mathrm{BF}$ & $37.6 \pm 5.1$ (men: 25.7; women: 22.6) & $37.6 \pm 5.1$ (men: 25.7; women: 22.6$)$ \\
\hline $\mathrm{WC}(\mathrm{cm})$ & $\begin{array}{c}93.5 \pm 12.2 \text { (men: } 101.7 \pm 9.1 \text {; women: } \\
85.3 \pm 9.5)\end{array}$ & $\begin{array}{c}93.0 \pm 11.6 \text { (men: } 101.0 \pm 7.9 \text {; women: } \\
85.0 \pm 9.0 \text { ) }\end{array}$ \\
\hline $\mathrm{HC}(\mathrm{cm})$ & $\begin{array}{c}97.5 \pm 8.2 \text { (men: } 100.3 \pm 8.5 \text {; women: } \\
94.7 \pm 8.5)\end{array}$ & $\begin{array}{l}97.5 \pm 8.2 \text { (men: } 100.3 \pm 8.5 \text {; women: } \\
94.7 \pm 8.5)\end{array}$ \\
\hline WHR & $\begin{array}{c}0.96 \pm 0.07 \text { (men: } 1.01 \pm 0.03 \text {; women: } \\
0.90 \pm 0.02)\end{array}$ & $\begin{array}{c}0.95 \pm 0.06 \text { (men: } 1.00 \pm 0.03 \text {; women: } \\
0.90 \pm 0.01)\end{array}$ \\
\hline RHR & $79.0 \pm 8.6$ & $77.5 \pm 9.2$ \\
\hline SBP (mmHg) & $104.5 \pm 9.7$ & $103.7 \pm 10.3$ \\
\hline $\mathrm{DBP}(\mathrm{mmHg})$ & $67.5 \pm 7.6$ & $67.5 \pm 7.6$ \\
\hline
\end{tabular}

BM: Body Mass; BMI: Body Mass Index; \%BF: Body Fat percentage; WC: Waist Circumference; HC: Hip Circumference; WHR: Waist to Hip Ratio; RHR: Resting Heart Rate; SBP: Systolic Blood Pressure; DPB: Diastolic Blood Pressure.

\subsection{Metabolic Parameters}

In ExT, plasma glucose levels increased $45 \mathrm{~min}$ following breakfast consumption $(\% \mathrm{z}=-2.20$; $p=0.028)$, immediately following exercise $(\% \mathrm{z}=-1.99 ; p=0.046)$ and $1 \mathrm{~h}$ following exercise $(\% \mathrm{z}=-1.99$; $p=0.046$ ) compared to baseline levels (before breakfast consumption). In CoT, plasma glucose levels were increased $45 \mathrm{~min}$ following breakfast consumption $(\% \mathrm{z}=-2.20 ; p=0.028)$ and immediately following rest ( $\% \mathrm{z}=-2.02 ; p=0.043)$ compared to baseline levels (before breakfast consumption). Moreover, pairwise comparisons showed that there was no significant difference in glucose levels at any time point between trials (Figure 1). Table 2 indicates time related changes with significant increases observed at $45 \mathrm{~min}$ post-meal following the exercise and control trials, immediately post-exercise/control 
and $1 \mathrm{~h}$ post-exercise. Moreover, no difference in glucose area under the curve (AUC) between trials or gender was observed (Figure 2).

\section{Glucose}

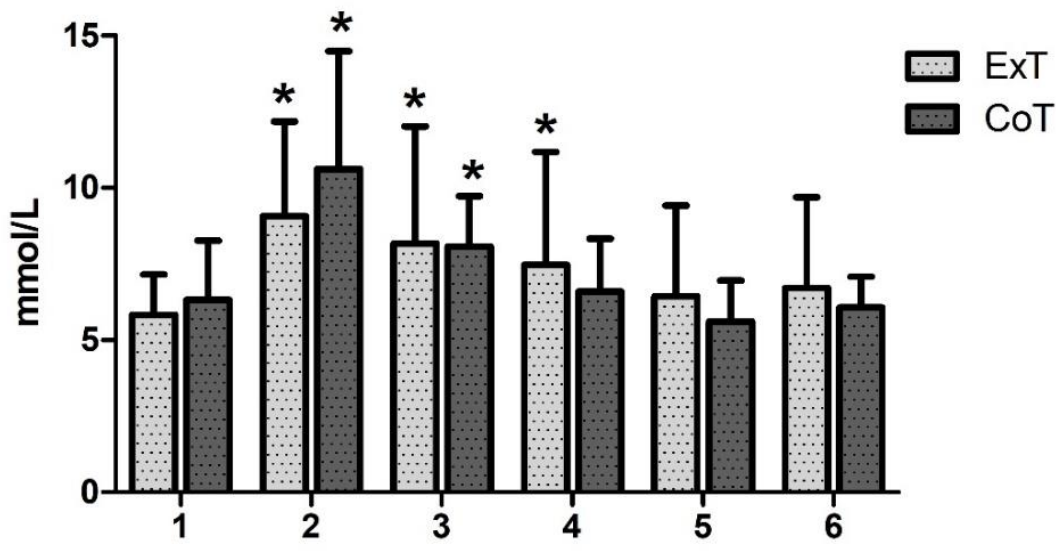

Figure 1. Changes in plasma glucose levels (mmol/L) following exercise (ExT) and control trial (CoT). Time points: (1) pre-meal; (2) 45 min post-meal (pre-exercise/control); (3) immediately post-exercise/control; (4) $1 \mathrm{hr}$ post-exercise/control; (5) $2 \mathrm{~h}$ post-exercise/control; (6) $24 \mathrm{~h}$ post-exercise/control. *Significant difference from (1) at the same trial.

Table 2. Changes in plasma glucose $(\mathrm{mmol} / \mathrm{L})$ levels (mean \pm SD) following exercise (ExT) and control trial (CoT). Time points: (1) pre-meal; (2) 45 min post-meal (pre-exercise/control); (3) immediately post-exercise/control; (4) $1 \mathrm{~h}$ post-exercise/control; (5) $2 \mathrm{~h}$ post-exercise/control; (6) $24 \mathrm{~h}$ post-exercise/control. * Significant difference from (1) at the same trial.

\begin{tabular}{lcc}
\hline Time point & ExT & CoT \\
\hline 1 & $5.83 \pm 1.33$ & $6.32 \pm 1.95$ \\
2 & $9.07 \pm 3.11^{*}$ & $10.62 \pm 3.87^{*}$ \\
3 & $8.17 \pm 3.85^{*}$ & $8.07 \pm 1.66^{*}$ \\
4 & $7.47 \pm 3.71^{*}$ & $6.60 \pm 1.73$ \\
5 & $6.43 \pm 2.99$ & $5.61 \pm 1.35$ \\
6 & $6.71 \pm 2.99$ & $6.07 \pm 1.01$ \\
\hline
\end{tabular}

\section{Glucose}

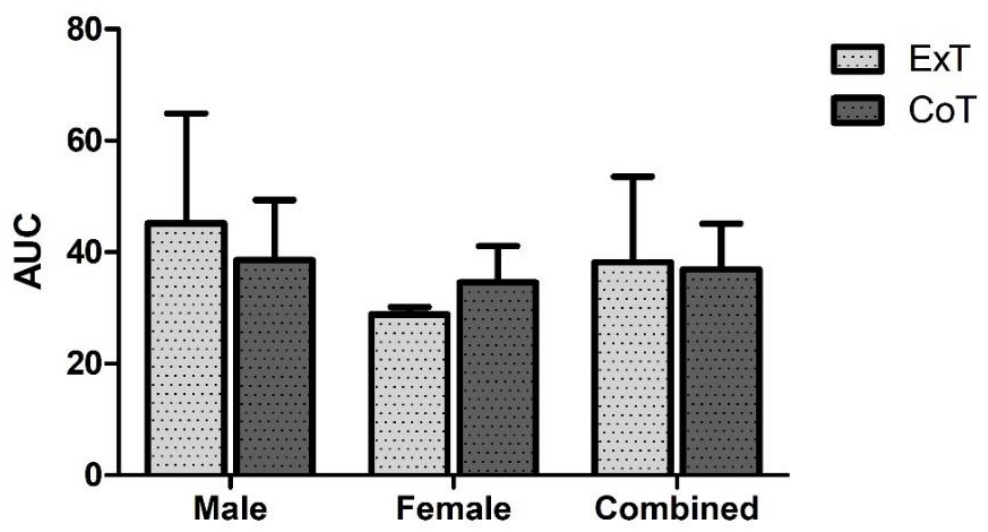

Figure 2. Glucose area under the curve $(\mathrm{AUC})$ in $\mathrm{mmol} / \mathrm{L} \times 24 \mathrm{~h}$ in exercise $(\mathrm{ExT})$ and control trial (CoT). 
Triglycerides, total cholesterol, HDL and LDL levels did not change at any time point and were similar in both conditions (Figure 3a-d). Moreover, no difference in triglycerides AUC between trials or gender was observed (Figure 4).
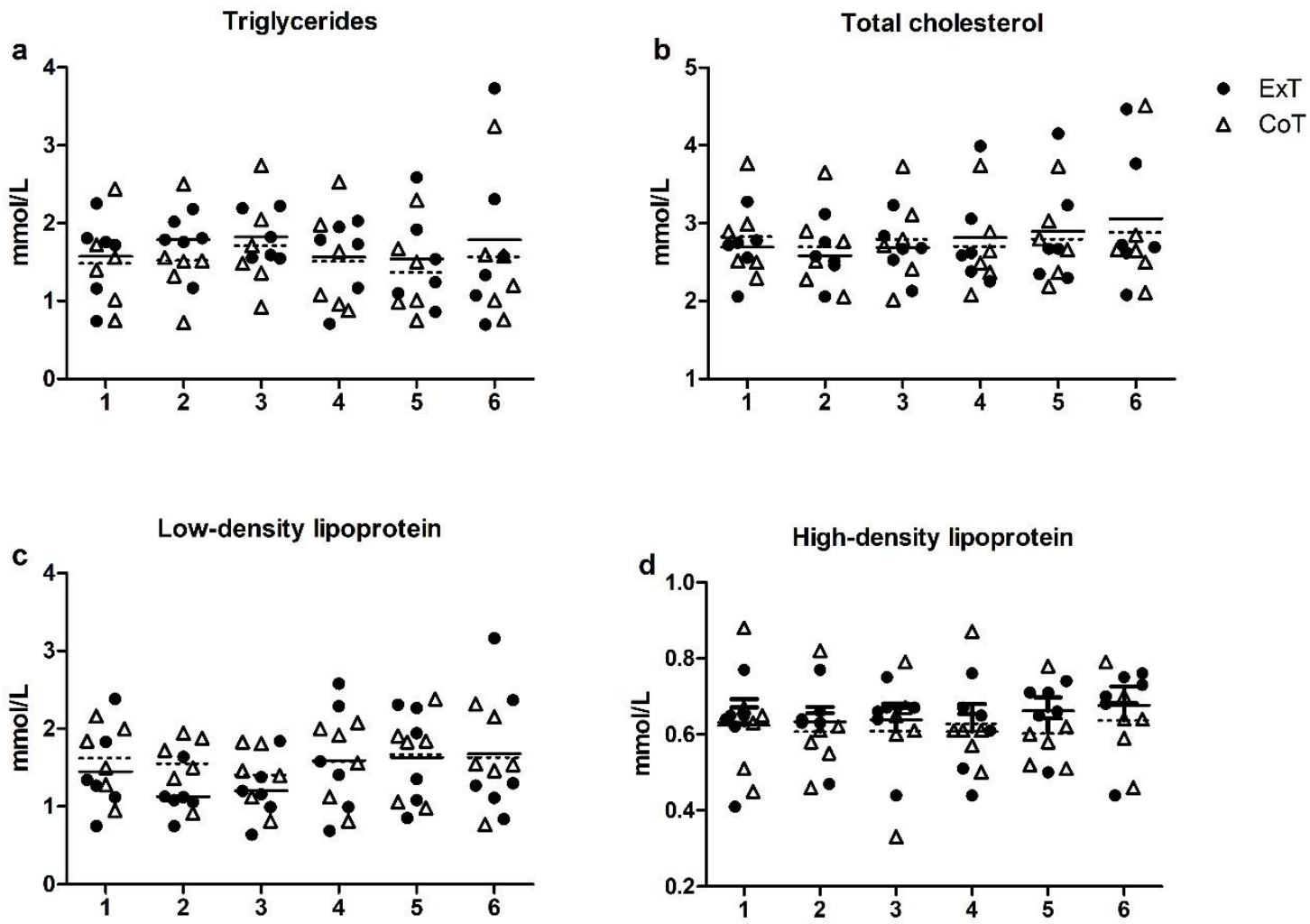

Figure 3. Changes in plasma lipid levels following exercise (ExT) and control trial (CoT): (a) Triglycerides; (b) Total cholesterol; (c) LDL: Low Density Lipoprotein; (d) HDL: High Density Lipoprotein. Time points: (1) pre-meal; (2) $45 \mathrm{~min}$ post-meal (pre-exercise/control); (3) immediately post-exercise/control; (4) $1 \mathrm{~h}$ post-exercise/control; (5) $2 \mathrm{~h}$ post-exercise/control; (6) $24 \mathrm{~h}$ post-exercise/control. Dotted lines represent the mean values of the Control trial.

\section{Triglycerides}

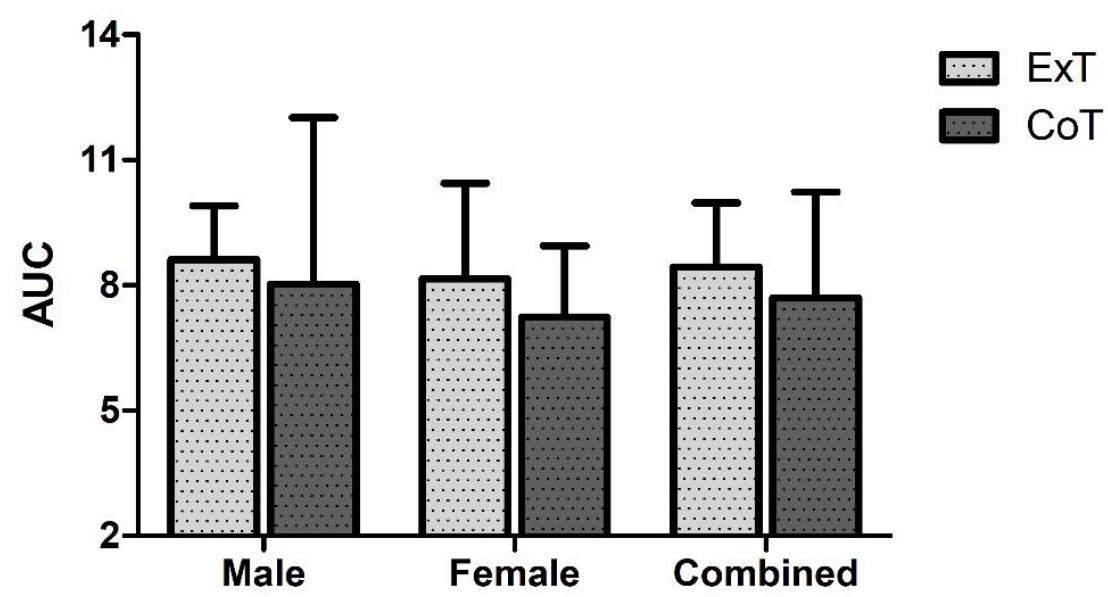

Figure 4. Triglycerides area under the curve (AUC) in $\mathrm{mmol} / \mathrm{L} \times 24 \mathrm{~h}$ in exercise (ExT) and control trial (CoT). 


\section{Discussion}

The main aim of this study was to examine whether postprandial resistance exercise can influence changes in blood glucose in prediabetic, BTM patients. Supplementary analyses of blood lipids were performed as these parameters are associated with glucose metabolism, DM and cardiovascular disease. To the authors' knowledge, this is the first study to test this hypothesis in this clinical population. Our findings suggest that an acute bout of postprandial (post-breakfast) resistance exercise is not a sufficient stimulus to (i) attenuate the blood glucose response, and (ii) change the lipids profile, throughout the subsequent 24-h post-exercise period.

Although research on the effects of resistance training on glycaemic control in type $2 \mathrm{DM}$ patients is scarce, there is some data suggesting that this modality could be beneficial [15]. Moreover, the positive effects of a single session of aerobic exercise (50\% maximum workload capacity) and a single session of resistance exercise (75\% 1RM) on the 24-h average blood glucose levels and the 24-h prevalence of hyperglycaemia were found to be similar [16]. In the present study, we did not observe any change in blood glucose levels throughout the subsequent 24-h post-exercise period. It is possible that prediabetic, BTM patients do not have similar responses to those of type 2 DM patients as the underlining pathophysiology could lead to different results. Moreover, the high level of SD observed could be explained by the small sample. The population examined in our study has very unique characteristics and many factors (i.e., medication, frequency of blood transfusion, comorbidities) can lead to different responses. Moreover, SD was higher in Ext at time points 2-6, indicating that these unique characteristics may have an important role in blood glucose responses to exercise for up to 24-h. Besides, it has been reported that BTM patients often manifest exercise intolerance and fatigue mediated by anaemia and iron-mediated cardiotoxicity [9]. Thus more research comparing the effects of resistance exercise in prediabetic, BTM patients and DM patients is warranted.

The timing of exercise relative to meal consumption may also play a role in glycaemic control. The limited available data indicate that postprandial exercise may be more beneficial than preprandial exercise in type $2 \mathrm{DM}$ patients [3]. Postprandial resistance exercise causes a greater reduction in glucose incremental area under the curve (iAUC) (reduction by 30\%) compared to preprandial resistance exercise (reduction by 18\%) [4]. In addition, postprandial resistance exercise improves triglyceride levels, another risk factor for cardiovascular disease in type 2 DM [4]. Thus, postprandial resistance exercise may be an effective means of better glycaemic control and lower risk of cardiovascular disease in individuals with abnormal glucose metabolism. Based on this, Borror et al. [3] proposed that resistance exercise should be performed following the largest meal of the day, 2 to 3 non-consecutive days per week, at intensities varying between $50 \%$ and $80 \%$ of $1 \mathrm{RM}$, working the major muscle groups (1-4 sets of 8-15 repetitions/exercise) [17,18]. In the present study, we used an acute resistance exercise protocol at an intensity of 70\% of 1RM and we found no change in blood glucose levels, glucose AUC, lipid levels or triglycerides AUC throughout the 24-h post-exercise period. The limited available data suggest that resistance exercise could have positive effects that are associated with increases in lean muscle mass and type II fibre type recruitment [15]. However, all aforementioned studies refer to acute postprandial exercise studies and it needs to be stated that it is of great importance to perform exercise training interventions to assess the long-term effects of exercise on not only the acute hyperglycaemia, but the long-term glycaemia control as well. Previous reports indicate that glycaemia in type $2 \mathrm{DM}$ males and females is different with males exhibiting higher impaired fasting glycaemia and women impaired glucose tolerance $[19,20]$. The results from this study do not coincide with the aforementioned reports since there were no differences between males and females neither at rest nor following the glycaemic load (AUC) at rest and after exercise. However, it needs to be stated here that the sample size for males $(n=3)$ and females $(n=3)$ is rather small, this constitutes a limitation of the study and concrete conclusions should be made with caution.

Postprandial hypertriglyceridemia has also been linked to increased risk of cardiovascular disease [21]. In this study, blood lipids (triglycerides, total cholesterol, HDL and LDL) did not change at any time point and were similar in both trials. This was also evident in the lack of difference in 
triglycerides AUC between trials. This may be explained by the fact that the breakfast provided was low in saturated fat. A meal high in saturated fat increases blood triglyceride levels as well as indices of oxidative stress and inflammation, resulting in a worsening of endothelial dysfunction, vasoconstriction and systolic blood pressure [22,23]. Therefore, exercise following lunch or dinner could be more beneficial in terms of cardiovascular health.

Patients with abnormal glucose levels are often diagnosed with Metabolic Syndrome (MS), a multiple set of risk factors that confer an additional cardiovascular risk [24]. In this study, it was shown that men were overweight and women were of normal weight according to BMI, and that both genders had central obesity according to the WC cut-point [14]. The participants were also prediabetic and had low HDL levels, meeting the criteria for metabolic syndrome set by the International Diabetes Federation. As already mentioned, BTM causes various complications due to anaemia and iron overload, and the presence of MS is an additional cardiovascular risk, rendering BTM patients a clinical population with unique characteristics. Therefore, BTM patients may not respond to exercise in a typical manner and long-term interventions are required.

In conclusion, postprandial resistance exercise, especially following the largest meal of the day, could be an effective means of glycaemic control and could lower the risk of cardiovascular disease in DM patients [3]. The results of the present study do not indicate that a bout of acute resistance exercise $45 \mathrm{~min}$ following breakfast is a sufficient stimulus to improve blood glucose lipid levels throughout the subsequent 24-h post-exercise period. Future studies comparing the acute and chronic effects of resistance exercise in prediabetic, BTM patients and DM patients are needed.

Author Contributions: Conceptualization, A.Z.J.; methodology, A.Z.J., K.G. and C.K.D.; validation, A.Z.J.; formal analysis, K.G.; investigation, K.G., A.S., C.K.D. and N.S.; resources, A.Z.J. and A.S.; data curation, K.G. and A.Z.J.; writing — original draft preparation, K.G.; writing-review and editing, A.S., I.G.F. and A.Z.J.; visualization, K.G., D.D. and A.S.; supervision, A.Z.J. and I.G.F.; project administration, A.Z.J. and A.S.; funding acquisition, A.Z.J. and I.G.F. All authors have read and agreed to the published version of the manuscript.

Funding: This research received partial funding from the Postgraduate Program of Study "Exercise and Health: Testing \& Prescription", Department of P.E. \& Sport Science, University of Thessaly, Greece.

Conflicts of Interest: The authors declare no conflict of interest.

\section{References}

1. World Health Organization. Definition and Diagnosis of Diabetes Mellitus and Intermediate Hyperglycemia: Report of a WHO/IDF Consultation; World Health Organization: Geneva, Switzerland, 2006; pp. 1-50.

2. Bansal, N. Prediabetes diagnosis and treatment: A review. World J. Diabetes 2015, 6, 296-303. [CrossRef] [PubMed]

3. Borror, A.; Zieff, G.; Battaglini, C.; Stoner, L. The Effects of Postprandial Exercise on Glucose Control in Individuals with Type 2 Diabetes: A Systematic Review. Sports Med. 2018, 48, 1479-1491. [CrossRef] [PubMed]

4. Heden, T.D.; Winn, N.C.; Mari, A.; Booth, F.W.; Rector, R.S.; Thyfault, J.P.; Kanaley, J.A. Postdinner resistance exercise improves postprandial risk factors more effectively than predinner resistance exercise in patients with type 2 diabetes. J. Appl. Physiol. 2015, 118, 624-634. [CrossRef] [PubMed]

5. Taher, A.T.; Saliba, A.N. Iron overload in thalassemia: Different organs at different rates. Hematology. Hematol. Am. Soc. Hematol. Educ. Program 2017, 2017, 265-271. [CrossRef]

6. Cario, H.; Holl, R.W.; Debatin, K.M.; Kohne, E. Insulin sensitivity and beta-cell secretion in thalassaemia major with secondary haemochromatosis: Assessment by oral glucose tolerance test. Eur. J. Pediatr. 2003, 162, 139-146. [CrossRef]

7. Halliwell, B.; Gutteridge, J.M.C. Role of free radicals and catalytic metal ions in human disease: An overview. Methods Enzymol. 1990, 186, 1-85.

8. Tiedge, M.; Lortz, S.; Drinkgern, J.; Lenzen, S. Relation between antioxidant enzyme gene expression and antioxidative defense status of insulin-producing cells. Diabetes 1997, 46, 1733-1742. [CrossRef]

9. Sohn, E.Y.; Kato, R.; Noetzli, L.J.; Gera, A.; Coates, T.; Harmatz, P.; Keens, T.G.; Wood, J.C. Exercise performance in thalassemia major: Correlation with cardiac iron burden. Am. J. Hematol. 2013, 88, 193-197. [CrossRef] 
10. ClinicalTrials.gov. US National Institutes of Health. Available online: http://www.clinicaltrials.gov (accessed on 14 January 2020).

11. Georgakouli, K.; Manthou, E.; Fatouros, I.G.; Deli, C.K.; Spandidos, D.A.; Tsatsakis, A.M.; Kouretas, D.; Koutedakis, Y.; Theodorakis, Y.; Jamurtas, A.Z. Effects of acute exercise on liver function and blood redox status in heavy drinkers. Exp. Ther. Med. 2015, 10, 2015-2022. [CrossRef]

12. Friedewald, W.T.; Levy, R.I.; Fredrickson, D.S. Estimation of the concentration of low-density lipoprotein cholesterol in plasma, without use of the preparative ultracentrifuge. Clin. Chem. 1972, 18, 499-502. [CrossRef]

13. Faul, F.; Erdfelder, E.; Lang, A.-G.; Buchner, A. G*Power 3: A flexible statistical power analysis program for the social, behavioral, and biomedical sciences. Behav. Res. Methods 2007, 39, 175-191. [CrossRef] [PubMed]

14. Alberti, K.G.; Zimmet, P.; Shaw, J. Metabolic syndrome-A new world-wide definition. A Consensus Statement from the International Diabetes Federation. Diabet. Med. 2006, 23, 469-480. [CrossRef] [PubMed]

15. Laughlin, M.H. Physical activity-induced remodeling of vasculature in skeletal muscle: Role in treatment of type 2 diabetes. J. Appl. Physiol. 2016, 120, 1-16. [CrossRef] [PubMed]

16. van Dijk, J.W.; Manders, R.J.; Tummers, K.; Bonomi, A.G.; Stehouwer, C.D.; Hartgens, F.; van Loon, L.J. Both resistance- and endurance type exercise reduce the prevalence of hyperglycaemia in individuals with impaired glucose tolerance and in insulin-treated and non-insulin-treated type 2 diabetic patients. Diabetologia 2012, 55, 1273-1282. [CrossRef]

17. Colberg, S.R.; Sigal, R.J.; Fernhall, B.; Regensteiner, J.G.; Blissmer, B.J.; Rubin, R.R.; Chasan-Taber, L.; Albright, A.L.; Braun, B.; American College of Sports Medicine, American Diabetes Association. Exercise and type 2 diabetes: The American College of Sports Medicine and the American Diabetes Association: Joint position statement. Diabetes Care 2010, 33, e147-e167. [CrossRef]

18. Colberg, S.R.; Sigal, R.J.; Yardley, J.E.; Riddell, M.C.; Dunstan, D.W.; Dempsey, P.C.; Horton, E.S.; Castorino, K.; Tate, D.F. Physical activity/exercise and diabetes: A position statement of the American Diabetes Association. Diabetes Care 2016, 39, 2065-2079. [CrossRef]

19. Mauvais-Jarvis, F. Gender differences in glucose homeostasis and diabetes. Physiol. Behav. 2018, 187, $20-23$. [CrossRef]

20. van Genugten, R.E.; Utzschneider, K.M.; Tong, J.; Gerchman, F.; Zraika, S.; Udayasankar, J.; Boyko, E.J.; Fujimoto, W.Y.; Kahn, S.E. American Diabetes Association GENNID Study Group. Effects of sex and hormone replacement therapy use on the prevalence of isolated impaired fasting glucose and isolated impaired glucose tolerance in subjects with a family history of type 2 diabetes. Diabetes 2006, 55, 3529-3535. [CrossRef]

21. Nordestgaard, B.; Benn, M.; Schnohr, P.; Tybjaerg-Hansen, A. Nonfasting triglycerides and risk of myocardial infarction, ischemic heart disease, and death in men and women. JAMA 2007, 289, 299-308. [CrossRef]

22. Blum, S.; Aviram, M.; Ben-Amotz, A.; Levy, Y. Effect of a Mediterranean meal on post-prandial carotenoids, paraoxonase activity and C-reactive protein levels. Ann. Nutr. Metab. 2006, 50, 20-24. [CrossRef]

23. Jakulj, F.; Zernicke, K.; Bacon, S.L.; van Wielingen, L.E.; Key, B.L.; West, S.G.; Campbell, T.S. A high fat meal increases cardiovascular reactivity to psychological stress in healthy young adults. J. Nutr. 2007, 137, 935-939. [CrossRef] [PubMed]

24. Sattar, N.; Gaw, A.; Scherbakova, O. Metabolic syndrome with and without creactive protein as a predictor of coronary heart disease and diabetes in the West of Scotland Coronary Prevention Study. Circulation 2003, 108, 414-419. [CrossRef] [PubMed]

(C) 2020 by the authors. Licensee MDPI, Basel, Switzerland. This article is an open access article distributed under the terms and conditions of the Creative Commons Attribution (CC BY) license (http://creativecommons.org/licenses/by/4.0/). 